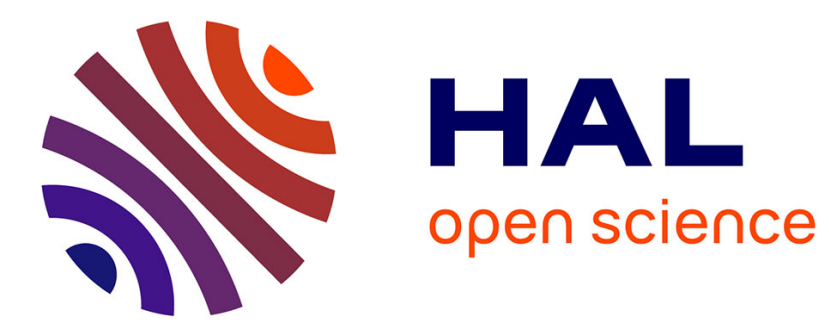

\title{
Modélisation et conception multiniveau de systèmes complexes : stratégie d'agentification des organisations
}

Gildas Morvan, Alexandre Veremme, Daniel Dupont, Daniel Jolly

\section{To cite this version:}

Gildas Morvan, Alexandre Veremme, Daniel Dupont, Daniel Jolly. Modélisation et conception multiniveau de systèmes complexes: stratégie d'agentification des organisations. Journal Européen des Systèmes Automatisés (JESA), 2009, 43 (4-5), pp.381-406. hal-00391949

HAL Id: hal-00391949

https://hal-univ-artois.archives-ouvertes.fr/hal-00391949

Submitted on 24 Sep 2021

HAL is a multi-disciplinary open access archive for the deposit and dissemination of scientific research documents, whether they are published or not. The documents may come from teaching and research institutions in France or abroad, or from public or private research centers.
L'archive ouverte pluridisciplinaire HAL, est destinée au dépôt et à la diffusion de documents scientifiques de niveau recherche, publiés ou non, émanant des établissements d'enseignement et de recherche français ou étrangers, des laboratoires publics ou privés. 


\title{
Modélisation et conception multiniveau de systèmes complexes : stratégie d'agentification des organisations
}

\author{
Gildas Morvan*, Alexandre Veremme ${ }^{*, * *}$, Daniel Dupont ${ }^{* *}$, Daniel Jolly ${ }^{*}$ \\ * Univ. Artois, UR 3926, \\ Laboratoire de Génie Informatique et d'Automatique de l'Artois (LGI2A), \\ F-62400 Béthune, France \\ ** HEI - Pôle Ingénierie et Sciences du Vivant, \\ F-59000 Lille, France
}

\section{Introduction}

Pour modéliser un système, il est nécessaire de disposer de données, même lacunaires, sur celui-ci. Dans le cas des systèmes complexes ${ }^{1}$, le modélisateur ne dispose que très rarement d'informations exhaustives sur le système à un niveau d'observation donné. Il est généralement confronté à un ensemble de données éparses, ne concernant que quelques aspects du système, de lois générales, i.e., relations entre propriétés, toutes choses étant égales par ailleurs. Après étude des données dont il dispose, deux possibilités s'offrent à lui : considérer le système comme un tout cohérent dont certaines propriétés présentent des régularités quantifiables ou qualifiables, ou comme un ensemble de parties en interrelations faisant émerger ces mêmes régularités. Le choix de l'une ou l'autre alternative implique l'utilisation de paradigmes de modélisation mutuellement inconsistants et repose généralement sur des raisons pratiques et/ou personnelles : résultats attendus, données disponibles, difficultés liées à l'implémentation, familiarité avec le paradigme, intuition, etc. Ainsi, même si le modélisateur a conscience que le système étudié est complexe, i.e., qu'il est à la fois tout, composé de soussystèmes, et partie d'un méta-système, il peut et souvent doit faire abstraction de telles considérations afin de construire un modèle opérationnel à partir des données et observations dont il dispose.

Un modèle holiste (i.e., qui considère le système comme un tout) permet de modéliser le comportement d'un système « en ne retenant que des structures ou

1. Dans le cadre de cet article, le mot complexe n'est pas à prendre au sens de « compliqué » mais se réfère à l'idée de nombreuses entités en interaction. La notion de système complexe est définie plus précisément dans la section 2.1 . 
des dynamiques globales ignorant ainsi en grande partie les phénomènes sousjacents et leurs interactions » [45]. Ce type de modèle décrit donc le comportement d'une propriété sans considérer les conditions " à l'intérieur du système ", qui permettent son émergence. D'autre part, un modèle émergent (e.g., un modèle centré individu, orienté agent ou encore basé sur un réseau d'automates cellulaires) permet de simuler comment le comportement global d'un système émerge à partir des interactions entre ses constituants (figure 1). Cet article sera centré sur le paradigme agent, celui-ci étant très général. Il n'impose en effet aucune contrainte sur l'architecture des entités représentées (c'est à la fois sa force et sa faiblesse) et permet donc de décrire tout système [37].

Ces deux approches, holiste et émergente, peuvent se révéler dans certains cas inadaptées à la modélisation ou à la conception de systèmes très complexes, évolutifs et donc nécessairement mal connus comme les systèmes vivants ou inspirés du vivant. Cela est particulièrement vrai lorsque le comportement du système dépend fortement de son contexte écosystémique et que celui-ci est très variable [9].

Ainsi, l'approche holiste ne permet pas, dans le cas général, de modéliser les conditions qui permettent d'appliquer telle ou telle loi, celles-ci ne pouvant être connues sans modéliser le système du point de vue de ses constituants. De plus, dans le cas des systèmes complexes, de tels modèles permettent généralement de comprendre le comportement de certaines propriétés prises indépendamment mais plus rarement la dynamique de l'ensemble des propriétés systémiques.

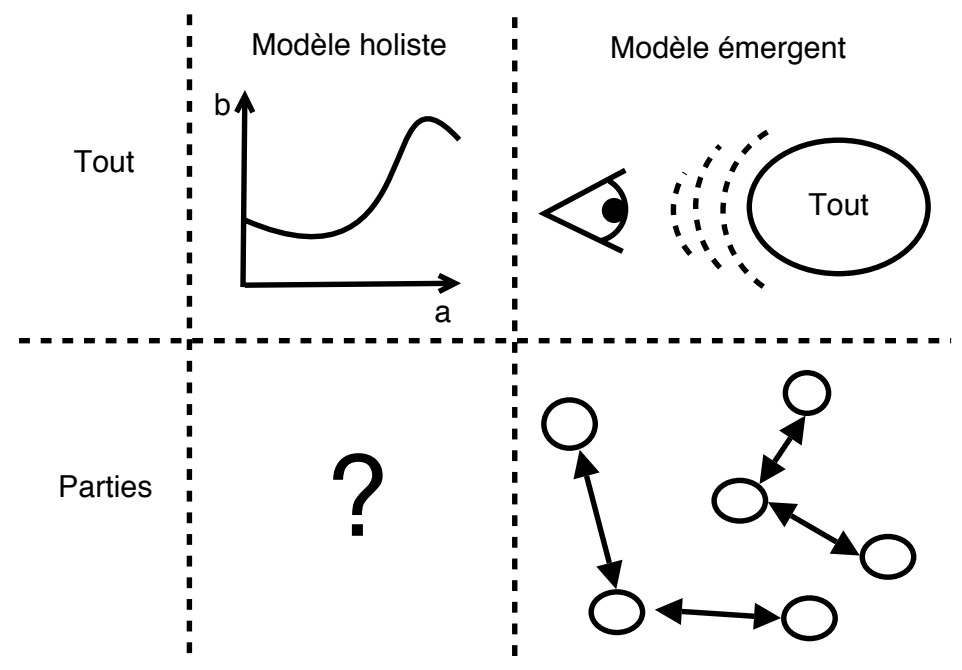

Figure 1. Approches holiste et émergente

La modélisation émergente répond, ou tout du moins a été conçue, pour répondre à ce problème. Cependant, modéliser de manière adéquate un système du point de vue de ses constituants suppose de modéliser de manière adéquate les constituants (objets, agents, environnement) ainsi que les interactions entre 
eux et l'environnement. Or, cela peut se révéler particulièrement difficile lorsque certains aspects de leurs comportements sont eux-mêmes particulièrement complexes, et donc difficiles à modéliser voire tout simplement à déterminer expérimentalement pour des raisons pratiques. Le modélisateur doit déterminer l'ensemble des comportements susceptibles d'expliquer le ou les phénomènes observés (hypothèses) et, à la suite d'un processus de validation de ces hypothèses, déterminer la ou les plus plausibles. On parle de raisonnement abductif ou « raisonnement en faveur de la meilleure explication » pour caractériser un tel processus de choix et de validation d'hypothèses [3]. Ce type de raisonnement étant par essence conjectural, la validité de la solution repose sur la qualité des méthodes de choix et de validation des hypothèses. Si dans un cadre formel et sur des problèmes jouets de telles méthodes ont été développées et validées, en pratique cela est bien plus difficile : les critères de validation d'une hypothèse peuvent être plus ou moins qualitatifs et subjectifs, le processus de validation d'une hypothèse est délicat et son exécution fastidieuse, le nombre d'hypothèses peut être important et en dernier ressort, le choix des hypothèses dépend de l'imagination plus ou moins fructueuse du modélisateur. Ainsi, la qualité de la solution produite ne peut être assurée et les modèles émergents peuvent finalement se révéler très mal adaptés et moins réalistes que leur homologues holistes.

Bien souvent d'ailleurs, l'utilisation de l'un de ces deux types de modélisation, holiste ou émergente, est également problématique pour la modélisation et le contrôle de systèmes de production. Ainsi, pour faire face à de nouvelles contraintes de fabrication (e.g., personnalisation en masse des produits, réduction des coûts de transport, traçabilité, etc.) [30], de nouvelles méthodes de pilotage de la production ont récemment vu le jour. Dans ce cadre, deux principales approches se distinguent : hiérarchique (que l'on pourrait rapprocher de l'approche holiste) et hétérarchique ${ }^{2}$ (que l'on pourrait rapprocher de l'approche émergente). La première considère généralement le système de contrôle de la chaîne de production comme une hiérarchie à plusieurs niveaux [2]. L'autre, inspirée des systèmes biologiques ${ }^{3}[40,38]$ ou basée sur le concept de produit actif et communicant (système de production holonique HMS [42]) ont mis le produit au centre de la chaîne logistique comme acteur interactif de son propre cycle de fabrication [35]. Des systèmes contrôlés par le produit [26] ont alors pu se développer grâce à l'arrivée de nouvelles technologies (RFID, GPS, etc.). Mais, les structures hiérarchiques apparaissent souvent trop rigides [41] et, à l'inverse, les méthodes émergentes, même si elles sont plus flexibles, peuvent difficilement prendre en compte l'ensemble des contraintes et règles comportementales imposées aux acteurs d'une chaîne logistique par son concepteur.

Ainsi, pour remédier à la « simplification de la complexité » des modèles holistes et au manque de fiabilité des modèles émergents, nous proposons dans cet article une méthode de modélisation et de contrôle des systèmes complexes, basée sur le paradigme agent, permettant d'unifier au sein d'un même modèle ces deux approches et donc de prendre en compte les connaissances du modélisateur

2. Une hétérarchie désigne une direction collégiale par opposition à la hiérarchie.

3. La notion de Biological Manufacturing System (BMS) est généralement employée pour désigner ce type de systèmes. 
aux différents niveaux d'observation et leurs relations. Cette méthode s'appuie sur le caractère organisationnel des systèmes et pose comme nécessaire la compréhension de la dynamique des organisations au sein d'un système complexe. Ainsi, des liens peuvent être établis avec les travaux traitant de la « réification de l'émergence » dans les modèles orientés agents [9]. Elle se distingue en revanche de la modélisation hybride dans laquelle des modèles microscopique et macroscopique sont liés par des relations de production-utilisation de données et dans laquelle chaque modèle est supposé définir adéquatement le système à un niveau d'observation donné [13].

L'approche proposée dans cet article consiste donc à modéliser, du point de vue des agents, la façon dont une organisation émerge à partir de leurs interactions et, du point de vue du système, les contraintes imposées aux agents par l'organisation ainsi que les propriétés émergentes. La dynamique des organisations se réalisant dans un système étant spécifiée à différents niveaux d'observation, cette méthode de modélisation est qualifiée de « multiniveau ».

Cette méthode a été initialement développée pour répondre à un problème de modélisation dans le domaine de la biologie [28]. Cependant elle peut être appliquée à la problématique du contrôle des systèmes complexes ; un exemple lié au routage de véhicules autoguidés est présenté dans la section 5.2. Mais avant tout, les concepts fondamentaux sont présentés, puis l'architecture de l'approche multiniveau proposée est détaillée.

\section{Concepts fondamentaux}

\subsection{Systèmes complexes et émergence}

Un système peut être défini comme une « unité globale organisée d'interrelations entre éléments, actions, ou individus »[27]. On parle de système complexe lorsque cette « unité globale » est composée de nombreux éléments interagissant localement et simultanément. Les systèmes complexes peuvent être le siège de processus de rétroaction, d'autoorganisation ou d'autopoï̀se (autoproduction), générant des émergences au niveau macroscopique.

Le terme « émergence » est fréquemment rencontré dans la littérature relative à de nombreux domaines comme la biologie, la philosophie [23], la sociologie [32], l'économie [10], la conception de système de production [39] et d'une manière générale, l'étude des systèmes complexes. Cependant, la définition même de la notion d'émergence pose un grand nombre de problématiques non résolues. Bien que dans la suite de cet article une définition volontairement « naïve » et générale, i.e., admise par l'ensemble des chercheurs s'intéressant à ce concept, est adoptée, il semble important de revenir sur les points importants permettant de spécifier cette notion.

Toute définition du concept d'émergence débute généralement par la citation de la maxime suivante, attribuée à Aristote : « la totalité est plus que la somme des parties»[4]. Cette maxime pose la non-équivalence entre les propriétés de «parties» ou composants pris indépendamment et les propriétés résultant 
de l'interaction de ces mêmes composants, formant un « tout » ou système. L'émergence est donc le processus par lequel un système acquiert de nouvelles propriétés ne pouvant être expliquées ou déduites par la seule connaissance de ses parties. Cette idée est formalisée par [5] de la manière (ici simplifiée) suivante : soit un ensemble de structures ${ }^{4} S_{1}$, une fonction $o b s_{1}$ permettant d'observer certaines propriétés de $S_{1}$. De plus, on suppose que les éléments de $S_{1}$ peuvent interagir. On pose $S_{2}=R_{1}\left(S_{1}\right)$ où $R_{1}$ est le résultat du processus d'interactions des structures de niveau 1 et $S_{2}$ est un ensemble de structures de niveau 2 . Soit $o b s_{2}$, une fonction d'observation permettant d'observer certaines propriétés de $S_{2}$. $P$ est une propriété émergente de $S_{2}$ si et seulement si $P \in o b s_{2}\left(S_{2}\right)$ mais $P \notin o b s_{1}\left(S_{1}\right)$. Cette définition est généralisable à tout ensemble de structures de $\operatorname{rang} N$.

Bien que cette définition pose le problème de l'existence de nouvelles propriétés « triviales ${ }^{5}$ » et qui ne devraient donc pas être considérées comme émergentes au sens où l'entendent par exemple [24], elle semble intéressante dans le cadre de notre problématique. En effet, elle intègre la problématique des différents niveaux d'observation d'un système, i.e., d'une hiérarchie de structures, et souligne l'importance de l'interaction dans le processus d'émergence.

Notons de plus que l'on distingue généralement deux types d'émergence : forte et faible. Les définitions de l'émergence forte sont relatives à l'apparition de nouvelles propriétés dans le système à un niveau de complexité donné, indépendamment de toute observation. En d'autres termes, l'apparition de ces propriétés ne peut (et ne pourra jamais) être prédite (et inversement expliquée) à l'aide de connaissances relatives aux propriétés du système à un niveau de complexité inférieur. D'autres définitions, telle celle de Baas, sont relatives à la difficulté pour un observateur, de qualifier les causes de cette apparition (et inversement de la prédire). La propriété observée est donc subjectivement considérée comme émergente. On parle alors d'émergence faible.

\subsection{Modélisation et simulation orientées agent}

Parmi les paradigmes de modélisation et de simulation des systèmes complexes, l'approche « orientée agent » se distingue par son applicabilité à de nombreux domaines et sa facilité de mise en ouvre [7]. Cette approche repose sur un ensemble de concepts et de méthodes développés dans le cadre des recherches sur les systèmes multi-agent (SMA) [15, 47]. [12] notent que dans le cadre de la modélisation/simulation orientée agent, trois instances du concept d'agent sont manipulées : les « agents réels » vus comme des entités pouvant être observées dans le système étudié (notion proche de celle d'acteur), les « agents conceptuels » constituant une formalisation des agents réels à l'aide des notions

4. Le terme « structure » est une abstraction pour désigner, par exemple, les notions de système, d'organisme, etc.

5. S'il est aisé de déterminer des exemples d' « émergence triviale », e.g., le fait qu'il soit possible de s'asseoir et de se maintenir à une certaine distance du sol sur une chaise mais que cela soit en revanche impossible sur les atomes, pris indépendamment, qui constituent cette même chaise, le problème de la définition de la notion de trivialité est tout sauf trivial. Le lecteur intéressé par ce problème peut se référer à [17]. 
relatives aux SMA (communications, interactions, etc.) et enfin les « agents informatiques » ou « agents computationnels», implémentations des agents conceptuels exécutables dans un environnement de simulation donné. Ce cadre méthodologique permet ainsi de concevoir des modèles émergents en manipulant des concepts ayant une sémantique bien définie.

Ainsi, un agent est un système capable de percevoir, et éventuellement de se représenter partiellement l'environnement dans lequel il est situé, d'agir dans celui-ci et de communiquer avec d'autres agents. De plus, un agent possède éventuellement des ressources propres et des objectifs à satisfaire. De nombreuses architectures d'agent (e.g., modulaire, de subsomption, compétitive, etc.) ont été développées ainsi que diverses méthodes et langages de communication (e.g., KQML [16]) adaptés à des problématiques variées. Un SMA est ici défini comme un ensemble d'entités $B$, situées dans un environnement $E$, avec $A \subset B$ un ensemble d'agents interagissant selon une certaine organisation ${ }^{6}$.

Un modèle orienté agent est un modèle émergent permettant de simuler les interactions d'individus dans un espace fini. L'activité de modélisation proprement dite consiste à concevoir un SMA qui, exécuté (simulé), permet de reproduire certaines régularités propres au système modélisé.

Implémenter efficacement un SMA n'est pas chose aisée : l'intégration des standards de communication, des mécanismes d'exécution adéquats, etc., sont autant de problèmes à résoudre pour le développeur. De nombreuses platesformes comme $\mathrm{JADE}^{7}$ ou MADKIT ${ }^{8}$ ont vu le jour afin de simplifier le développement, le déploiement et l'exécution de SMA. De plus, certaines plates-formes, comme NETLOGO ${ }^{9}$ ou TURTLEKIT [25], sont dédiées à la simulation et permettent au non-spécialiste d'implémenter et d'exécuter facilement un modèle orienté agent. En revanche, ces plates-formes imposent des contraintes fortes sur l'architecture des agents et leur exécution; ainsi, le choix de l'une ou l'autre plate-forme peut être un problème en soit [31].

\section{Organisation multiniveau d'un système}

Une organisation est définie par Edgar Morin comme «l'agencement de relations entre composants ou individus qui produit une unité complexe ou système, dotée de qualités inconnues au niveau des composants ou individus » [27]. L'organisation au sein d'un système permet l'émergence de nouvelles propriétés mais en revanche, elle contraint le comportement des éléments qui la constituent. Si l'on considère par exemple une organisation sociale, ses contraintes propres pourraient être définies comme l'ensemble des lois, coutumes, habitudes sociales qui la régissent et ses propriétés émergentes seraient sa culture, sa science, etc.

Dans les modèles organisationnels et méthodologies de conception de systèmes multi-agent centrées sur les organisations, les propriétés et contraintes

6. Cette problématique est traitée dans la section suivante.

7. http ://jade.tilab.com

8. http ://madkit.org

9. http ://ccl.northwestern.edu/netlogo/ 
propres à une organisation ne sont généralement pas énoncées spécifiquement et ne sont pas considérées comme des notions spécifiant l'organisation mais comme des propriétés émergentes. Notons cependant que dans le modèle MOISE, la notion de rôle est exploitée pour « contraindre les comportements individuels des agents »[19].

Plus généralement, ces modèles permettent de structurer l'activité des agents en utilisant des concepts comme les groupes ou les rôles. Par exemple, une organisation sera arbitrairement décrite par l'ensemble des rôles que les agents peuvent prendre dans un groupe, les interactions possibles entre rôles, etc. [14]. L'organisation est spécifiée à un niveau d'observation donné.

Cependant d'un point de vue pratique, les propriétés émergentes et les contraintes propres à une organisation donnée peuvent être observées et donc connues. Il semble donc intéressant d'intégrer cette connaissance dans les modèles émergents. Pour conserver le caractère émergent du modèle, cela suppose également d'intégrer les conditions permettant à cette organisation de naître, de se perpétuer et de mourir. Ainsi, une stratégie d'agentification du concept d'organisation est proposée.

\subsection{Agentification d'une organisation}

Le comportement des constituants d'un système (agents, objets) dépend des propriétés émergentes de celui-ci. Le tout, dépendant des parties, rétroagit donc sur les parties, celles-ci devenant dépendantes du tout. Cette relation de codépendance entre le tout et les parties a été étudiée par des chercheurs de différents horizons [27, 43] et a semble-t-il inspiré les concepteurs de systèmes multi-agent [15] mais n'a pas débouché sur une réelle remise en cause des paradigmes de modélisation. Cet article propose d'adopter une vision multiniveau des systèmes et donc des organisations se réalisant en eux et par eux.

Cette approche, qui considère qu'une organisation, une fois créée, possède une « vie » propre, suppose de considérer une organisation, non plus seulement comme une structure, mais également comme un agent. Un tel agent est appelé agent-organisation et un agent pouvant intégrer une organisation, agent-entité. Un agent-organisation doit être capable d'une communication « interne », i.e., avec les agents-entité constituant l'organisation, et 《 externe », i.e., avec d'autres agents-organisation. Son comportement dépend à la fois de ses constituants (qui spécifient les conditions dans lesquelles certaines propriétés émergent), des autres agents-organisation et bien sûr, de la dynamique propre à l'organisation (modélisation des contraintes et des propriétés émergentes). Notons que différents types d'agents-entité et d'agents-organisation peuvent interagir au sein d'un même système.

Ces notions d'agent-entité et d'agent-organisation ne sont en aucun cas absolues mais relatives, encore une fois, au niveau d'observation du système. Bien que les idées d'agent-organisation et de hiérarchie d'agents Entité et Organisation soient assez proches des concepts d'holon et d'holarchie [21, 1], dans cet article un vocabulaire purement « agent » sera employé. En effet, un agent-organisation est essentiellement défini par un cycle de vie particulier qui conditionne son exis- 
tence même (section suivante). Toutefois, dans un premier temps, pour faciliter la compréhension de la méthode et son implémentation, ne seront considérés que des systèmes observés à deux niveaux, macroscopique et microscopique, correspondant aux agents-organisation et agents-entité.

Il ne s'agit pas ici de soutenir un point de vue plutôt qu'un autre sur la nature des organisations ou l'organisation de la nature, mais de proposer une solution pratique aux problèmes que rencontrent le modélisateur de systèmes complexes, partiellement connus à divers niveaux d'observation, et le concepteur de systèmes artificiels, à la structure et aux objectifs complexes et évolutifs. Ainsi, un agent-organisation peut être vu comme un agent « virtuel », c'est-àdire n'ayant pas d'équivalent dans le système étudié, au niveau d'observation des constituants et de l'émergence de nouvelles propriétés, et réel au niveau d'observation considérant le système comme un tout.

La dynamique des organisations dans un système complexe peut être chaotique, stochastique, en un mot difficile à déterminer. Ainsi, les conditions dans lesquelles de nouvelles propriétés émergent peuvent être, elles aussi, difficiles à déterminer si l'on ne connaît pas, ou si l'on ne prend pas en compte le comportement des constituants du système. Dans cette approche, il n'est pas nécessaire de modéliser totalement ces comportements (il a été dit que cela pouvait présenter certaines difficultés, notamment pratiques), mais il est en revanche nécessaire de modéliser les interactions entre les agents. Ce propos peut sembler paradoxal, les relations entre constituants étant justement « le terreau» d'une organisation. Il faut donc clarifier la notion assez floue de «modélisation des interactions ». Il s'agit de la modélisation de l'ensemble des actions réciproques susceptibles de modifier le comportement des autres agents [15]. La stratégie de déplacement d'agents cherchant à s'éviter tout en voulant atteindre un but est un exemple typique d'interaction qui sera traité dans la section 5.2.

Cette méthode s'appuie donc sur deux aspects des systèmes : l'interaction et l'organisation, et situe ces deux concepts à des niveaux différents du point de vue de l'observateur et du modélisateur : aux niveaux microscopique et macroscopique (figure 2). La notion de "conditions nécessaires » à l'existence d'une organisation a été évoquée précédemment. Ces conditions portent sur certaines propriétés du système et permettent à un agent-organisation de déterminer si une propriété particulière peut émerger et comment.

\subsection{Cycle de vie d'un agent-organisation}

D'un point de vue pratique, un agent-organisation est créé par des agentsentité lorsque ceux-ci constatent que l'état de leurs relations est compatible avec les conditions de naissance de l'organisation qu'il représente. L'agent-organisation peut par la suite évoluer (e.g., en intégrant de nouveaux agents, en développant de nouvelles contraintes et propriétés émergentes) ou mourir en fonction de l'état des relations entre agents. Les agents-entité intègrent les connaissances liées à la naissance des organisations qu'ils peuvent constituer et les agents-organisation les connaissances liées à la vie et à la mort de l'organisation qui leur est propre.

Le cycle de vie de l'agent-organisation peut donc se résumer aux activités 

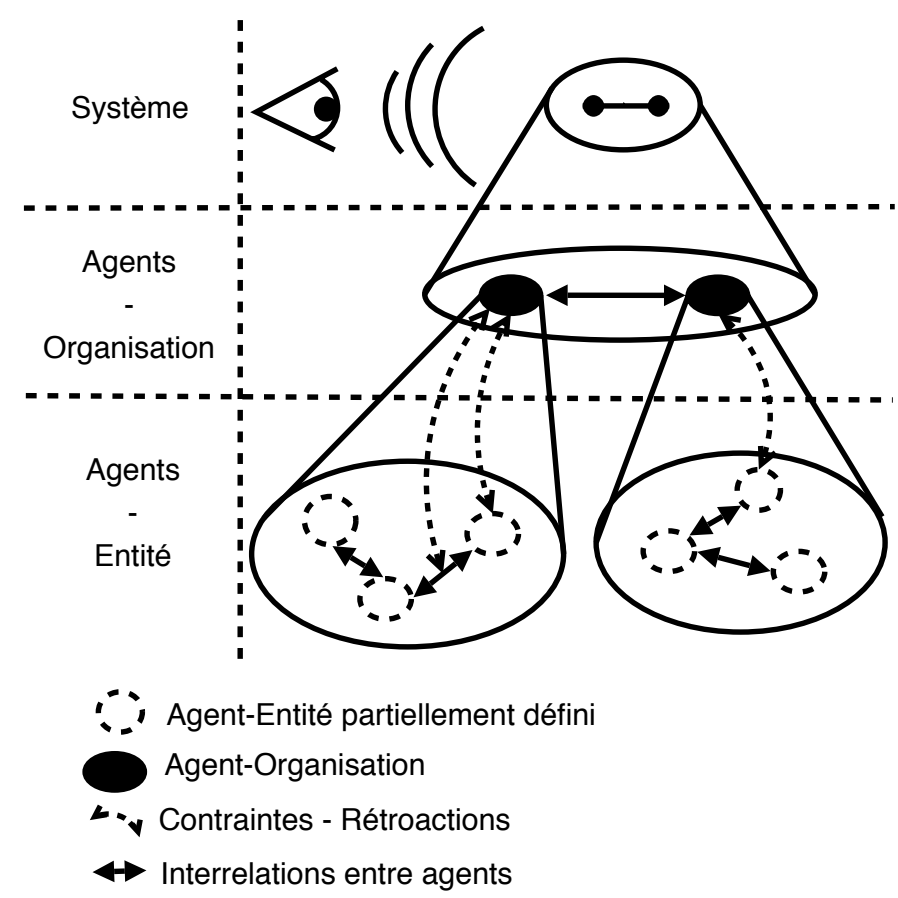

Figure 2. Concepts manipulés aux niveaux microscopique et macroscopique dans la méthode de modélisation proposée

suivantes : (1) vérifier que les contraintes définissant l'organisation sont vérifiées (en d'autres termes, vérifier que l'organisation existe encore); (2) calculer les propriétés émergentes en fonction de l'état des relations entre agents au sein de l'organisation et entre l'agent-organisation et les autres agents-organisation; (3) communiquer le résultat de ces calculs aux agents concernés (figure 3). Le calcul d'une propriété émergente est intimement lié à l'application. Ainsi, dans l'exemple développé dans la section 5.1, la valeur la propriété émergente considérée (la température) est une fonction du nombre d'agents-entité (larves d'insectes) dans l'organisation (un agrégat).

L'agent-organisation n'est pas seulement un simple superviseur ou observateur du système comme peut le laisser supposer la définition du cycle de vie de l'agent-organisation et l'utilisation du terme « communiquer ». En effet, communiquer la valeur d'une propriété permet à l'agent-organisation de rétroagir sur le comportement des agents-entité (si leurs comportements dépendent de cette propriété). 


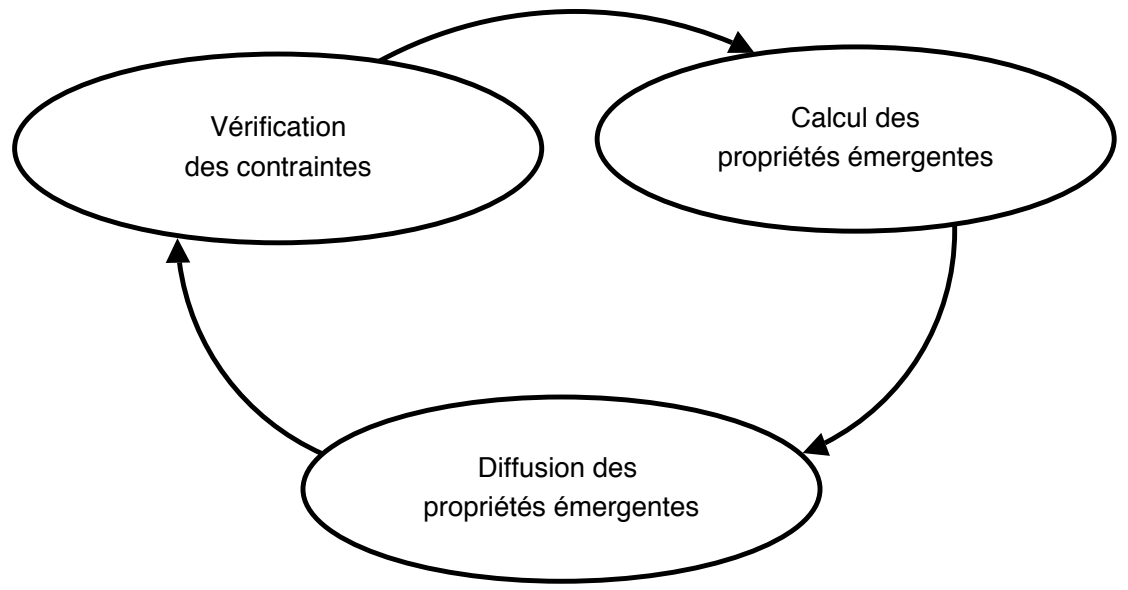

Figure 3. Cycle de vie d'un agent-organisation

\section{Implémentation et problématiques}

La méthode de modélisation, introduite dans la section précédente, vise à résoudre un ensemble de problèmes pratiques mais peut, par ailleurs, en poser d'autres liés à l'implémentation d'un tel modèle. Certains d'entre eux sont abordés dans cette section et des pistes de réflexion, basées sur des travaux antérieurs, sont proposées pour les résoudre. Par la suite, une stratégie d'implémentation, basée sur des méthodes de communication stigmergique, est indroduite.

\subsection{Problématiques d'implémentation}

\subsubsection{Synchronisation des agents}

Le cycle de vie d'un agent-organisation, a été décrit sans avoir été relié au cycle de vie des agents-entité composant l'organisation. Pour implémenter un système basé sur une telle méthode, il faut spécifier comment ces cycles de vie peuvent se synchroniser. Il semble pertinent de s'inspirer du concept de « société de modèles » permettant de faire collaborer des modèles hétérogènes dans un même système [8]. Dans cette approche, les modèles sont « agentifiés » et les agents-modèle ainsi constitués produisent et utilisent des résultats locaux ou « artefacts » pour construire des patterns, c'est-à-dire des compositions d'informations permettant de comprendre le comportement du système. Un agent-modèle exécute le modèle qu'il encapsule tant qu'il dispose des données nécessaires pour le faire.

Il s'agit d'un mode de synchronisation sans doute trop « basique » car dans l'approche multiniveau, le problème de la synchronisation des cycles de vie des différents agents vient du fait que le comportement de l'organisation est coproduit par l'ensemble des agents-entité qui la composent et l'agent-organisation. Ce problème semble particulièrement crucial dans le cadre de systèmes s'exé- 
cutant en temps réel et pour lesquels une discrétisation du temps n'est pas possible.

\subsubsection{Efficacité des simulations}

Le principal problème lié à l'efficacité d'exécution d'un tel modèle est la quantité de messages potentiellement échangés pour observer (monitorer) le système. En effet, l'agent-organisation doit pour calculer ses propriétés émergentes, connaître l'état de l'organisation et donc l'observer. Les recherches visant à élaborer des méthodes efficaces de monitoring portent essentiellement sur des systèmes dans lesquels des agents cognitifs sont physiquement distribués et communiquent intensivement pour atteindre leurs objectifs [20]. Dans le cas de simulations multi-agent, les problématiques sont différentes et des travaux ont montré que des approches organisationnelles permettent un monitoring efficace [18].

L'approche proposée dans [29] est basée sur le concept de report based monitoring dans lequel chaque agent reporte son état à un superviseur [46]. Cependant, faut-il réellement observer chaque agent pour calculer la valeur d'une propriété émergente? En d'autres termes, l'information à observer est-elle « véhiculée » par tous les agents ou par un sous-ensemble d'entre eux ? Cette méthode d'observation est basée sur la notion de groupe. Un groupe est défini comme un ensemble d'agents qui contiennent les informations nécessaires au calcul d'une propriété. Les agents adhèrent volontairement aux groupes. Ils doivent donc s'autoobserver pour déterminer s'ils doivent rejoindre, quitter ou rester dans un groupe. Un système à base de règles, permettant de faciliter la conception de la phase d'autoobservation, a été proposé. Un ensemble de règles, représentant une observation particulière et spécifié par le modélisateur/observateur, est évalué à chaque pas de simulation. Un agent appartient à un groupe si et seulement si l'ensemble des règles définissant le groupe est positivement évalué. Dans le cadre de la méthode de modélisation proposée dans cet article, les règles d'autoobservation pourraient être intégrées dynamiquement dans les agents-entité par des agents-organisation et non par le modélisateur.

\subsection{Une stratégie d'implémentation stigmergique}

Dans cette section, une stratégie d'implémentation du modèle multiniveau basée sur la notion de stigmergie est introduite. La stigmergie est une méthode de communication indirecte utilisant l'environnement comme medium [6]. Les individus communiquent en modifiant localement, dans l'espace et le temps, leur environnement. D'abord étudiée en entomologie (les fourmis et les termites notamment utilisent ce type de communication), la stigmergie a été utilisée dans des algorithmes d'optimisation, basés sur le comportement de fourragement des fourmis [11]. Nous nous plaçons ici dans le cadre de SMA pour lesquels le temps et l'espace sont discrétisés et l'exécution des agents est synchronisée. Cela signifie que l'environnement est découpé en cellules, et qu'à chaque pas d'exécution, un ordonnanceur autorise chaque agent à exécuter une partie de son cycle de vie. 
Concrètement, et dans le cadre des SMA, les agents communiquent en diffusant des signaux et en déposant des marques dans l'environnement. Un signal est une propriété environnementale se propageant selon une loi qui lui est propre. Une marque est un signal ne se propageant pas et au temps de vie éventuellement limité. Par exemple, il est possible d'associer à chaque cellule un ensemble de variables d'environnement. Chaque variable d'environnement possède trois paramètres : sa valeur, un coefficient de diffusion et un coefficient d'évaporation. Le coefficient de diffusion représente la proportion de la valeur de la variable d'environnement qui sera répartie équitablement entre les voisins de la cellule au pas d'exécution suivant. Le coefficient d'évaporation représente la proportion de la valeur de la variable d'environnement (après diffusion) qui sera perdue par la cellule au pas d'exécution suivant. Les agents doivent bien sûr disposer d'effecteurs leur permettant de manipuler des variables d'environnement. Le comportement d'une variable d'environnement s'inspire de celui d'une phéromone et permet, par exemple, de modéliser la dynamique d'un système complexe sous la forme d'un réseau d'automates cellulaires. Il est à noter que le comportement des variables d'environnement peut être modifié en intégrant des notions comme la saturation.

Cette stratégie d'implémentation repose sur l'existence d'un signal ou d'une agrégation de signaux et de marques permettant d'induire localement la ou les propriétés responsables d'une émergence à un niveau d'observation plus élevé. Ces signaux peuvent représenter certaines réalités du système étudié, e.g., un champ de potentiels, ou peuvent être introduits artificiellement dans le modèle.

Soit $O$, l'ensemble des organisations auxquelles un agent-entité peut appartenir et $F_{O}$ un ensemble de fonctions conditionnant l'existence d'organisations de $O$ (ces fonctions sont appelées « fonctions de conditionnement »), tels qu'il existe une application surjective de $O$ vers $F_{O}{ }^{10}$. Cependant, pour simplifier la notation, dans la suite de cet article nous considérerons que cette application est bijective (une fonction de $F_{O}$ ne conditionne l'existence que d'une seule organisation de $O$ ).

Ainsi, formellement, on pose l'existence d'un ensemble de fonctions conditionnant la création des différents agents-organisation, i.e.,

$$
\forall o \in O, \forall c \in E, f_{o}: V(c) \mapsto\{\top, \perp\}
$$

où $f_{o}$ est la fonction de conditionnement de l'organisation $o, E$ représente l'ensemble des cellules de l'environnement et $V(c)$ représente l'ensemble des valeurs des variables d'environnement de la cellule $c$. Les fonctions de conditionnement sont implémentées dans chaque agent-entité; en revanche, l'évaluation de ces fonctions dépend de l'état des variables d'environnement de la cellule dans laquelle se trouve l'agent et non de l'état de l'agent lui-même.

Cependant, il existe un risque que plusieurs agents-organisation soient créés pour une même organisation. Ce problème peut être résolu en analysant de manière systématique l'ensemble des cellules de l'environnement, ce qui peut être

10. La notion de «type d'organisation » pourrait être utilisée pour désigner l'ensemble des organisations partageant une même fonction de conditionnement. 
problématique si le nombre de cellules est élevé. En revanche, un algorithme d'analyse de l'environnement plus efficace peut être utilisé si l'on pose la condition suivante : une organisation ne peut s'exprimer que dans une partie continue de l'environnement, i.e., dans un continuum de cellules ou « îlot». Cette condition peut paraître restrictive si l'on se limite à l'idée de l'environnement comme « espace géographique ». En effet, l'environnement peut représenter de manière plus générale l'espace topologique ${ }^{11}$ dans lequel interagissent les agents ou en d'autres termes, le « contexte d'interaction » des agents, pouvant intégrer des dimensions immatérielles, e.g. relatives aux caractéristiques sociales ou « psychiques » des agents (objectifs, intentions, etc.). Formellement, cette condition peut s'écrire sous la forme :

$$
\begin{aligned}
& \forall o \in O, \forall c \in E \text {, si } f_{o}(V(c))=\top \\
& \text { alors, } \forall c^{\prime} \in E-\operatorname{Ilot}_{o}(c), f_{o}\left(V\left(c^{\prime}\right)\right)=\perp
\end{aligned}
$$

où $\operatorname{Ilot}_{o}(c)$ représente l'ensemble des cellules au voisinage étendu ${ }^{12}$ de $c$ et $c$ elle-même, tel que,

$$
\forall c^{\prime \prime} \in \operatorname{Ilot}_{o}(c), f_{o}\left(V\left(c^{\prime \prime}\right)\right)=\top
$$

La fonction d'analyse de l'environnement (analyseEnvironnement) exécutée séquentiellement par chaque agent-entité est présentée dans l'algorithme 1. Dans cet algorithme, $m_{o}$ est une marque (variable d'environnement ne se diffusant pas) à la durée de vie limitée à un pas d'exécution, représentant le fait qu'une cellule ait été analysée durant le pas courant. Si la fonction de conditionnement retourne $\top$ alors l'algorithme est exécuté récursivement pour l'ensemble des cellules au voisinage de $c$ (Voisins $(c))$.

Lorsqu'un agent-entité exécute analyseEnvironnement $(c, o)$, si la fonction retourne un ensemble de cellules non vide, alors l'agent crée l'agent-organisation correspondant à l'organisation o et lui transmet l'ensemble retourné ainsi que son identifiant (algorithme 2). L' agent-organisation pourra ainsi rétroagir sur les agents faisant partie de l'organisation qu'il représente en modifiant les variables d'environnement des cellules où se « situe » l'organisation. L'agent-entité créant un agent-organisation prend le rôle de représentant de l'agent-organisation. Aux pas d'exécution suivants, l'agent-organisation réifiant l'organisation $o$ se substitue à son représentant, dans sa phase de vérification des contraintes propres à l'organisation, pour l'analyse de l'environnement (mais seulement pour l'organisation $o)$. Si analyseEnvironnement $(c, o)$, où $c$ représente la cellule du représentant de l'agent-organisation, retourne l'ensemble vide, alors ce dernier « se suicidera ». De plus, la stratégie d'ordonnancement suivante est imposée : les agents-organisation exécutent leur cycle de vie prioritairement aux agents-entité.

Cependant, ce mode de vérification des contraintes peut être peu efficace, notamment s'il existe un turn-over organisationnel important, i.e., si des agents

11. Un espace topologique est un ensemble muni d'une structure permettant de définir la notion de voisinage d'un élément de cet ensemble.

12. « Le voisin de mon voisin est mon voisin. » 
Entrée : La cellule $c$ sur laquelle est situé l'agent exécutant la fonction

Entrée : Une organisation o que l'agent-entité peut intégrer

Sortie : $\operatorname{Ilot}_{o}(c)$, l'ensemble des cellules où se « situe » l'organisation

1: $\operatorname{Ilot}_{o}(c) \leftarrow \emptyset$

2: $\mathbf{s i} m_{0} \notin V(c)$ alors

$V(c) \leftarrow V(c) \cup m_{o}$

si $f_{o}(V(c))=\top$ alors

$\operatorname{Ilot}_{o}(c) \leftarrow\{c\}$

pour tout $c^{\prime} \in V$ oisins $(c)$ faire

Ilot $_{o}(c) \leftarrow \operatorname{Ilot}_{o}(c) \cup$ analyseEnvironnement $\left(c^{\prime}, o\right)$

8: $\quad$ fin pour

9: $\quad$ fin si

10: fin si

Algorithme 1. Fonction analyseEnvironnement : détermination de l'ensemble des cellules dans lequel se "situe " une organisation

intègrent et quittent fréquemment l'organisation. Ainsi une fonction de vérification plus élaborée (existenceOrganisation), présentée dans l'algorithme 3 peut être utilisée. Cette fonction exécute récursivement analyseEnvironnement pour chacune des cellules non vides de $\operatorname{Ilot}_{o}(c)$, i.e., contenant au moins un agententité. Elle met à jour l'identifiant du représentant et l'ensemble des cellules où se « situe » l'organisation si l'existence de l'organisation est confirmée. Dans le cas contraire, l'agent-organisation se suicide. L'utilisation de ce mode de vérification des contraintes peut se traduire par une efficacité moindre si le coût d'exécution moyen de la fonction existenceOrganisation est supérieur au coût de destruction puis de création d'un agent-organisation.

Entrée : La cellule $c$ sur laquelle est situé l'agent-entité exécutant la fonction

Entrée : L'ensemble $O$ des organisations que l'agent-entité peut intégrer

1: pour tout $o \in O$ faire

Ilot $_{o}(c) \leftarrow$ analyseEnvironnement $(c, o)$

si $\operatorname{Ilot}_{o}(c) \neq \emptyset$ alors

Créer un nouvel agent-organisation pour $o$ avec $I_{l o t}(c)$ et l'identifiant de l'agent-entité exécutant la fonction comme paramètres

5: fin si

6: fin pour

Algorithme 2. Fonction creationAgentsOrganisation : création d'agentsorganisation si cela est nécessaire

Cette stratégie d'implémentation reposant sur la stigmergie pour, d'une part déterminer si une organisation existe, et d'autre part déterminer comment cette 
Entrée : L'identifiant a du représentant de l'agent-organisation au pas d'exécution précédent

Entrée : La cellule $c$ sur laquelle est situé le représentant de l'agent-organisation exécutant la fonction

Entrée : L'organisation o que représente l'agent-organisation

Entrée : $\operatorname{Ilot}_{o}(c)$, l'ensemble des cellules où se situait l'organisation au pas d'exécution précédent

Sortie : L'identifiant a du représentant de l'agent-organisation au pas d'exécution courant

Sortie : Ilot $t_{o}(c)$, l'ensemble des cellules où se situe l'organisation au pas d'exécution courant

1: Ilot $_{o}^{\prime}(c) \leftarrow$ analyseEnvironnement $(c, o)$

2: si $\operatorname{Ilot}_{o}^{\prime}(c)=\emptyset$ alors

3: $\quad$ si Ilot $_{o}(c)-\{c\}=\emptyset$ alors

4: $\quad$ Se suicider

5: $\quad$ sinon

6: $\quad$ Choisir une cellule non vide $c^{\prime}$ de $\operatorname{Ilot}_{o}(c)$. Soit $a^{\prime}$ l'identifiant de l'un des agents-entité présents dans $c^{\prime}$.

7: $\quad a, \operatorname{Ilot}_{o}(c) \leftarrow$ existenceOrganisation $\left(a^{\prime}, c^{\prime}, o, \operatorname{Ilot}_{o}(c)-\{c\}\right)$

8: $\quad$ fin si

9: sinon

10: $\quad \operatorname{Ilot}_{o}(c) \leftarrow \operatorname{Ilot}_{o}^{\prime}(c)$

11: fin si

Algorithme 3. Fonction existenceOrganisation : vérification de l'existence de l'organisation

organisation rétroagit sur ses constituants, permet de modéliser efficacement, i.e., en limitant au maximum les informations échangées entre agents, les différentes phases de la « vie » d'un agent-organisation : naissance, vie et mort. Dans le cadre d'un langage de programmation objet comme Java ${ }^{T M}$, les fonctions présentées dans cette section peuvent être facilement découplées du code des différents agents eux-même, en utilisant des méthodes « statiques », i.e., ne dépendant pas du contexte d'exécution des agents.

\section{Illustrations}

Deux illustrations du concept de modélisation multiniveau sont présentées dans cette section. La première a trait à la biologie tandis que la seconde propose une ébauche de solution au problème du routage de véhicules autoguidés dans un environnement complexe et incertain. 


\subsection{Application à l'entomologie médico-légale}

L'idée originelle de ce travail vient d'un problème lié à la modélisation du comportement thermique des masses de larves de diptères nécrophages. Ces larves sont le matériel de base de l'entomologie médico-légale, dont l'une des applications est la détermination de la date de la mort d'un individu, en fonction des insectes nécrophages retrouvés sur le corps. Pour dater la mort d'une personne, l'expert entomologiste cherche à déterminer le taux de développement accumulé par des insectes au moment de la découverte du cadavre. Ce taux est principalement dépendant de la température ressentie par les insectes au cours de leur vie [34]. Pour simplifier, plus l'environnement est chaud, plus l'insecte se développe rapidement. Cependant, sauf dans le cas d'un environnement contrôlé, il est extrêmement difficile de connaitre la température ressentie par un insecte : celle-ci est généralement fonction de la température ambiante, des caractéristiques thermiques du cadavre mais aussi du comportement grégaire des larves.

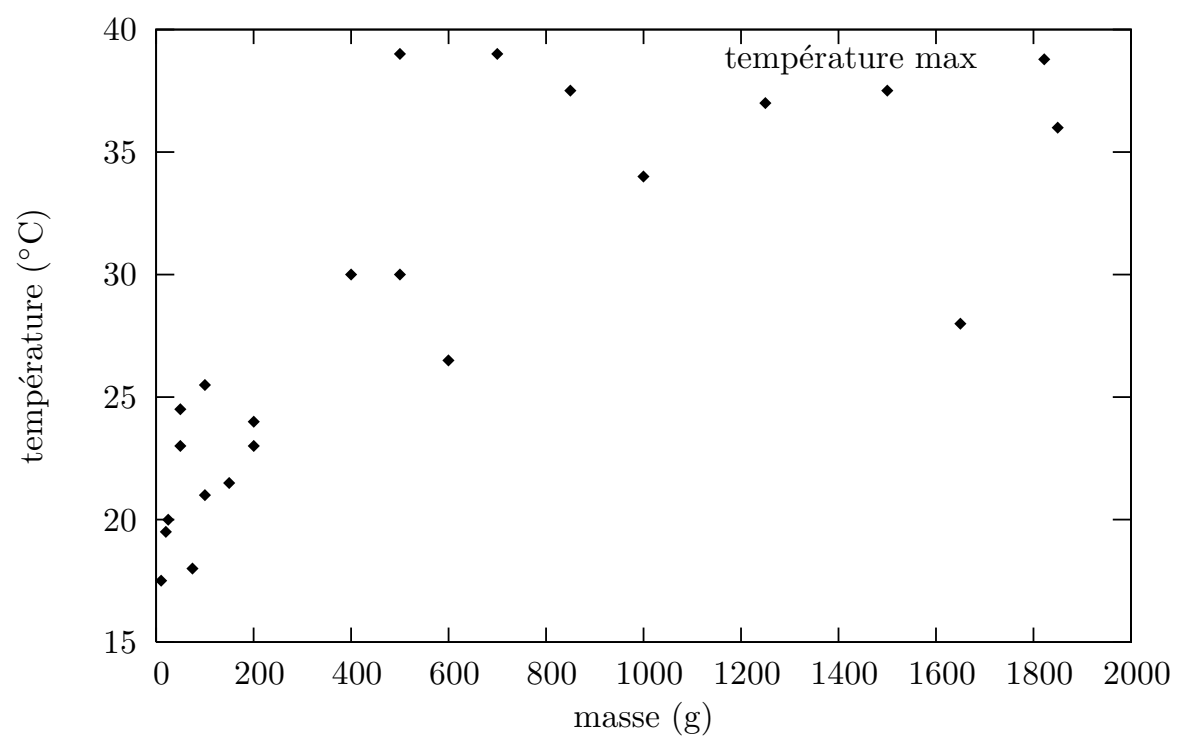

Figure 4. Température maximale observée dans des agrégats

Cette grégarisation est à l'origine d'un dégagement de chaleur, signalé dans de nombreux articles comme une source importante d'erreur dans l'estimation de l'intervalle post-mortem (e.g. [22]). La température à l'intérieur d'une masse de larves peut en effet être de plusieurs dizaines de degrés supérieure à celle de l'environnement et pourtant aucune méthode ne permet aujourd'hui de prendre en compte ce phénomène. Expérimentalement, il est en effet extrêmement difficile de mesurer la quantité de chaleur émise par une unique larve mais cette grandeur est quantifiable à l'échelle des masses de larves. C'est ce constat expérimental qui a motivé la mise en place de la méthode de modélisation multiniveau 
proposée dans cet article.

Ainsi, dans le cadre de cette méthode, les comportements thermiques ne sont plus spécifiés au niveau de l'individu (un agent-entité) mais de l'agrégat (agent-organisation). La dynamique des agrégats (naissance, vie, mort) est définie à partir des interactions entre larves, et de l'agrégat lui-même à partir des connaissances que l'on a de telles organisations (contraintes liées à l'accès à la nourriture, augmentation de la température et diffusion de la température en fonction de la masse et de la topologie de l'agrégat, dégradation de l'environnement, etc.). Le modèle a été implémenté selon la méthode présentée dans la section 4.2. Cependant, la fonction de vérification des contraintes présentée dans l'algorithme 3 n'a pas été utilisée.

Il a ainsi été possible de mesurer la quantité de chaleur dégagée par les agrégats en fonction de leur masse (figure 4) et de reproduire un dégagement similaire dans les simulations (figure 5). Sur la figure 5, nous observons la température ressentie par six larves choisies aléatoirement dans un agrégat. Cette température correspond à la température du cadavre tant que l'agrégat n'est pas formé (jusqu'à l'heure 9). Une fois l'agrégat constitué, une dynamique thermique apparaît durant laquelle la température se stabilise autour de $35{ }^{\circ} \mathrm{C}$. Cette dynamique s'éteint après 57 heures, lorsque les larves quittent l'agrégat qui se dissout alors.

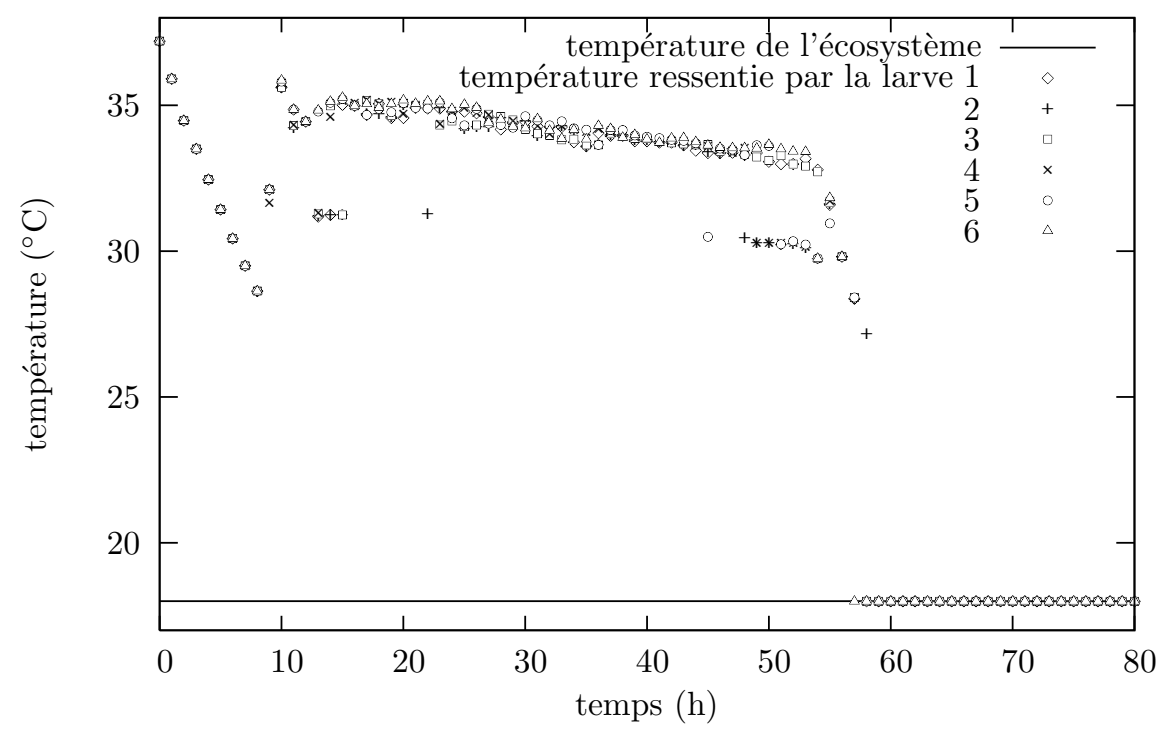

Figure 5. Température ressentie par 6 agents-entité choisis aléatoirement

Même si ce modèle doit encore être affiné et validé pour être utilisé dans le cadre d'enquêtes criminelles, il constitue d'ores et déjà un outil de simulation intéressant et fonctionnel : des scénarios complexes intégrant plusieurs milliers d'agents-entité de différents types, i.e., représentant des insectes de différentes 
espèces, comme celui présenté ci-dessus, peuvent être simulés efficacement. Le lecteur intéressé pourra se référer à $[29,28,44]$ pour plus de détails sur les méthodes mises en œuvre dans le cadre de cette application.

\subsection{Application au routage de véhicules autoguidés}

Pour illustrer ce propos au niveau industriel, nous nous plaçons dans le cadre couramment étudié $[33,40,36]$ du routage des véhicules à guidage automatique (AGV) ou véhicules autoguidés. Dans cette section, une implémentation de l'architecture multiniveau, ainsi que ses apports potentiels pour de telles problématiques, sont présentés.

\subsubsection{Présentation du contexte}

Un véhicule autoguidé est une unité mobile de manutention à déplacement automatique. En général, spécifique aux objets qu'il manipule, un AGV peut se mouvoir dans tout type d'environnement et ses modes de guidage sont variables (e.g., filoguidage, optoguidage, guidages laser, magnétique, etc.).

Les systèmes industriels intégrant ce type de véhicule sont habituellement décissionnellement centralisés et les comportements des AGV déterministes. Ces véhicules n'y ont aucune capacité de coopération et d'autonomie, même si quelques expériences ont déjà permis de les rendre interactifs. Bien qu'il puisse sembler naturel d'établir un système de contrôle hiérarchique dans des systèmes (entreprises) eux-même organisés hiérarchiquement, [39] soulignent les difficultés que rencontrent ces systèmes dans des environnements complexes et incertains (e.g., conjoncture économique peu prévisible, variabilité des besoins, etc.). De plus, les perturbations possibles à tous les niveaux de la chaîne, ainsi que les réactions correspondantes à déclencher sont difficilement anticipables et/ou modélisables. Et, une maintenance évolutive, même élémentaire (e.g., ajout, modification, suppression de ressources, etc.), est peu évidente à mettre en œuvre [42].

Ces systèmes présentent des problèmes de fiabilité et de robustesse qui peuvent être solutionnés par des architectures plus réactives basées sur l'idée de contrôle hétérarchique. Les relations hiérarchiques sont transformées en relations d'égal à égal et les AGV acquièrent plus d'autonomie. Plusieurs travaux, souvent inspirés du vivant [39], ont permis de mettre en place de telles structures. Mais l'« indépendance » des AGV n'est généralement pas propice à l'utilisation d'informations globales et des conflits locaux sont susceptibles d'apparaître, pour lesquels une résolution n'est pas aisée. En effet, la somme des objectifs de chacun des AGV n'est pas nécessairement égale au but du système global, pouvant être en constante évolution. Enfin, même s'ils sont basés sur les principes d'autoorganisation et d'apprentissage, ces systèmes de contrôle ne permettent pas toujours de garantir les performances minimales du système global lors de situations de crise ou imprévisibles [42]. 


\subsubsection{Modèles existants}

Une grande majorité des modèles de contrôle de véhicules autoguidés est basée sur le principe de contrôle hiérarchique. E.g., [33] proposent que l'environnement soit équipé de capteurs (e.g., des caméras) reliés à un système central capable de calculer les chemins optimaux (en prenant en compte les congestions par exemple) et de retransmettre ces informations aux véhicules concernés. Cependant, les algorithmes de résolution proposés ne prennent pas en compte l'ensemble des données du problème.

Dans [36], des capteurs sont directement positionnés sur les AGV mais le système de contrôle central est quant à lui inspiré des systèmes biologiques (ou Biological Manufacturing Systems) et plus particulièrement du comportement de fourragement des fourmis. Les AGV sont assimilés à des fourmis se déplaçant selon un gradient de phéromones. Il est possible d'effectuer des émulations (i.e., des simulations informatiques à vitesse accélérée) pour déterminer les chemins optimaux de chacun des AGV. Les AGV reçoivent alors leur « plan de route » et si l'un d'eux perçoit un problème, une nouvelle phase d'émulation est exécutée pour réorganiser le routage. On pourrait parler de contrôle semi-hétérarchique pour désigner ce type d'approche car bien que la planification du routage soit centralisée, les AGV ont un rôle d'« observateur actif ».

L'inspiration du vivant se retrouve également dans les diverses formes de contrôle hétérarchique. [38] proposent une approche stigmergique du routage des AGV en utilisant des champs magnétiques pour implémenter dans un système réel des « variables d'environnement » (principe des phéromones). Ainsi, contrairement aux approches précédentes, il n'existe pas de chemin prédéterminé : les AGV sont totalement autonomes dans les choix de leurs trajets. Pour s'orienter, les AGV sont attirés par les champs magnétiques émis par les ateliers qu'ils doivent desservir et émettent des champs répulsifs afin d'éviter les collisions et favoriser une bonne répartition des tâches. C'est l'existence de ces champs et la sensibilité des AGV à ceux-ci qui caractérisent le contrôle hétérarchique. Ainsi, un AGV disponible et percevant un champ attractif le remonte jusqu'à la source et empêche en parallèle ses semblables de faire de même, en émettant un champ répulsif. Cela permet, en théorie, d'éviter d'avoir plusieurs AGV affectés pour une même tâche. Cependant, des conflits peuvent émerger entre AGV et les performances du système peuvent en souffrir. De même, dans le pire des cas, les AGV peuvent rester bloqués, provoquant des congestions devant être réglées par un agent (typiquement un opérateur) extérieur. Pour résoudre ce problème, Ueda propose d'introduire des AGV à rationalité limitée, dont le comportement est partiellement aléatoire. Le système doit cependant être calibré afin de déterminer le nombre optimal d'AGV semi-rationnels à introduire. En effet, l'introduction d'un nombre trop important d'AGV de ce type peut dégrader considérablement les performances globales du système. 


\subsubsection{Approche multiniveau}

Une perspective d'implémentation de l'approche multiniveau est présentée dans cette section. À la frontière entre les approches hiérarchique et hétérarchique, la méthode proposée consiste à donner de l'autonomie aux AGV tout en permettant la création d'agents-organisation capables de régir le fonctionnement du système, ou d'une de ses parties, lors de l'apparition de perturbations. Nous nous plaçons dans le cadre de travail proposé par [38] et décrit ci-dessus : le routage d'AGV rationnels se déplaçant dans des champs de potentiels attractifs et répulsifs. Ainsi, en mode normal, les AGV sont autonomes et peuvent reconnaître et desservir les ateliers en toute indépendance. En mode dégradé, lorsqu'une modification survient (e.g., un problème lors du déplacement d'un véhicule, une panne sur une machine, une modification des besoins de production, etc.), un agent-organisation est créé et tente alors de rétroagir de manière automatique et globale pour optimiser le fonctionnement du système.

L'exemple de l'apparition et de la résolution de congestions dans un tel système est considéré. La fonction conditionnant la création des agents-organisation peut être définie comme suit : si un véhicule autoguidé (agent-entité) se voit dans l'impossibilité d'accéder à son point de destination au bout d'un temps $\tau$ (où $\tau$ est un seuil pouvant être défini de manière empirique ou par apprentissage) alors il déclenche la création d'un agent-organisation. Le problème de la synchronisation des cycles de vie des agents-entité (section 4.1.1) se pose dans un tel environnement temps réel. Un système de gestion des priorités pourrait être envisagé pour pallier cela. Cet agent-organisation, physiquement représenté par l'AGV l'ayant créé, va alors émettre un champ magnétique répulsif prédominant tous les autres champs de l'environnement local. Proche du fonctionnement des phéromones d'alarme de certains insectes [48], ce champ aura pour effet de faire fuir les AGV de la congestion. La puissance de l'émission de ce signal d'alarme peut être définie en fonction du nombre d'AGV impliqués dans la congestion. Ceux-ci chercheront simplement à s'échapper du champ « d'alarme » dans une direction choisie aléatoirement. L'agent-organisation se suicidera lorsque tous les AGV seront hors de portée du champ et qu'ils ne seront plus dans un état bloqué. L'AGV ayant créé l'agent-organisation reprend alors son fonctionnement normal en tant qu'agent-entité (figure 6).

Cette brève illustration permet déjà de mettre en valeur les possibilités de l'approche de contrôle multiniveau dans le cadre de chaînes de production. Il est en effet envisageable de considérer autant d'agents-organisation qu'il y aura de types d'agents-entité différents (i.e., toute machine pouvant être considérée comme un agent) et donc de pouvoir définir différents agents-organisation susceptibles d'interagir dans l'environnement. De plus, il sera également possible de considérer tout produit comme un agent-entité et de générer des agentsorganisation gérant le cycle de vie des produits de cette organisation. Enfin, cette structure semble particulièrement adaptée dans le cas de chaînes de productions dans lesquelles les produits sont fortement personnalisés (mass customization), où un agent-organisation pourrait, par exemple, augmenter ou atténuer de manière artificielle les champs d'attraction ou de répulsion d'agents-entité ayant 
des besoins plus ou moins importants. Des travaux plus approfondis devraient confirmer la flexibilité et la robustesse de l'approche dans le cas de modifications (volontaires ou subies) de la production.

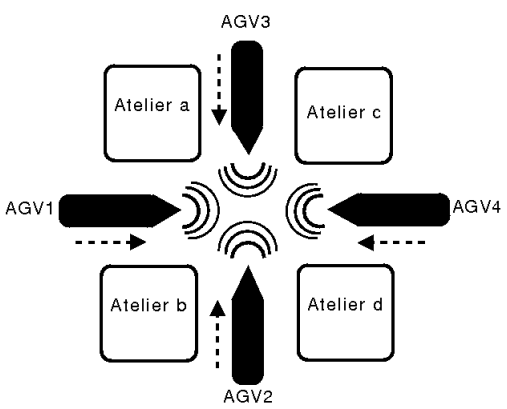

Naissance de la congestion

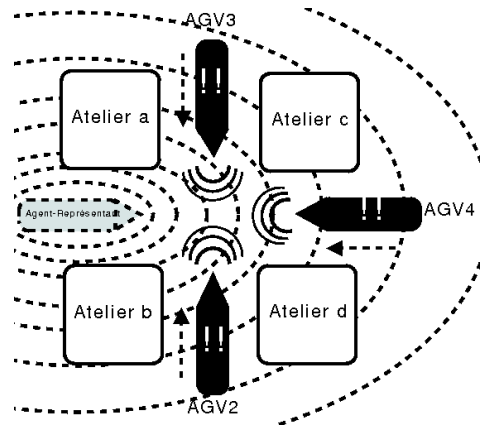

Création d'un agent-organisation, émission d'un signal répulsif

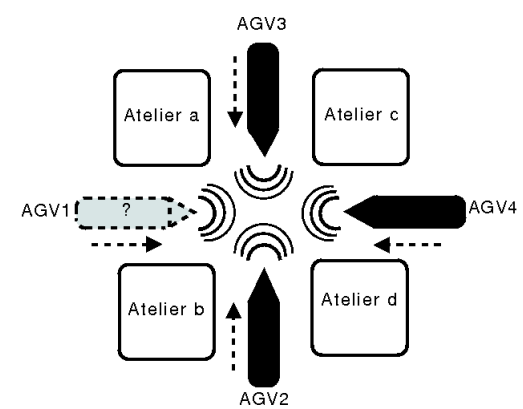

Détection de la congestion par l'AGV1

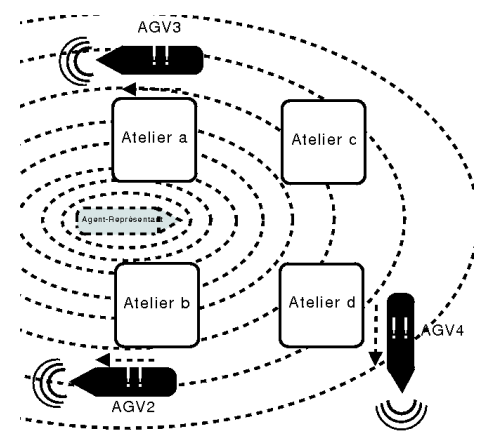

Résolution de la congestion

Figure 6. Illustration de l'approche multiniveau dans le cadre du routage de véhicules autoguidés

\section{Conclusion et travaux futurs}

Cet article propose une méthodologie adaptée à la modélisation, la conception et le contrôle de systèmes complexes considérés à différents niveaux d'observation, macroscopique (tout/système) et microscopique (parties/constituants). Le niveau macroscopique est représenté par des agents, appelés agents-organisation, qui : (1) calculent les nouvelles propriétés émergeant des interrelations entre les parties et des relations entre agents-organisation; et (2) imposent aux parties un certain nombre de contraintes. Ces procédures de calcul et contraintes sont définies explicitement durant la phase de modélisation. Le niveau microscopique est représenté par des agents, appelés agents-entité, dont le comportement n'est pas nécessairement totalement défini. Seuls : (1) les comportements et proprié- 
tés individuels permettant aux agents-entité d'interagir, et (2) les conditions de création d'une organisation doivent être impérativement définis.

L'objectif de cette méthode est de permettre au modélisateur d'utiliser dans un même modèle des données provenant de différents niveaux d'observation du système. Elle se distingue de l'approche « société de modèles » mais pourrait s'en nourrir. Dans cette approche, plusieurs modèles, concernant par exemple différents aspects du système, s'exécutent simultanément et communiquent leurs résultats à travers des structures spécialisées pour former un résultat d'ensemble cohérent. De même, elle n'est pas incompatible avec les modèles organisationnels existants mais offre un regard différent sur la notion d'organisation.

Dans le cadre de l'application présentée dans la section 5.1, les résultats obtenus sont déjà très prometteurs. D'un point de vue pratique, il serait intéressant d'implémenter l'approche multiniveau que nous proposons dans le cadre du routage de véhicules autoguidés sur une plate-forme de simulations et de comparer ses performances à celles de l'approche proposée par exemple par [38]. D'autres exemples d'application pourraient être envisagés dans le cadre des systèmes de production inspirés par le vivant (e.g., stockage de produits chimiques, contrôle de l'humidité du bois, etc.). Le développement de stratégies d'implémentation adaptées à des environnements temps réel (contrairement à l'approche stigmergique présentée dans cet article) permettrait d'appliquer cette méthode à de nombreux cas industriels. Plus généralement, il serait souhaitable de proposer un modèle d'implémentation générique et idéalement conçu, par exemple, comme une surcouche au modèle organisationnel AGR (agent-groupe-rôle) utilisé dans la plate-forme MadKit.

Enfin, cette méthode présente un certain nombre de problèmes pratiques qui se substituent à ceux qu'elle entend résoudre : synchronisation des différents agents " de différents niveaux », efficacité des simulations, et sans doute d'autres. Il est pourtant indispensable de vouloir résoudre des problèmes méthodologiques même si cela entraîne des difficultés d'implémentation. En effet, la complexité toujours croissante des systèmes artificiels et l'importance cruciale de mieux comprendre et maîtriser la complexité des systèmes naturels imposent le développement de nouvelles méthodes de modélisation et de raisonnement permettant d'acquérir une connaissance non mutilée de nos environnements technologiques et naturels.

\section{Remerciements}

Ce travail est financé par le Ministère de la Recherche et la Fondation Norbert Ségard. Les auteurs tiennent à remercier tout particulièrement Philippe Kubiak (LAGIS - Ecole centrale de Lille), Gilles Goncalves (LGI2A - Université d'Artois), Damien Charabidze et Benoît Bourel (Laboratoire d'entomologie Institut de médecine légale de Lille) pour leur soutien. Merci également aux relecteurs anonymes pour leurs remarques constructives et leurs encouragements. 


\section{Références}

[1] E. Adam. Modèle d'organisation multi-agent pour l'aide au travail coopératif dans les processus d'entreprise : application aux systèmes administratifs complexes. PhD thesis, Université de Valenciennes, 2000.

[2] J. S. Albus, C. R. McLean, A. J. Barbera, and M. L. Fitzgerald. Hierarchical control for robots in an automated factory, 1983.

[3] Atocha Aliseda-Llera. Seeking explanations : abduction in logic, philosophy of science and artificial intelligence. PhD thesis, Stanford University, Department of Computer Science, 1998.

[4] Aristote. La métaphysique. Flammarion, 2008.

[5] N. Baas. Emergence, hierarchies and hyperstructures. Artificial Life, 3 :515-537, 1992.

[6] E. Bonabeau. Artificial life, special issue on stigmergy, volume 5. MIT Press, Cambridge, 1999.

[7] E. Bonabeau. Agent-based modeling : methods and techniques for simulating human systems. Proceedings of the National Academy of Sciences, 99(3) :7280-7287, 2002.

[8] Stephane Bonneaud, Pascal Redou, and Pierre Chevaillier. Pattern oriented agent-based multi-modeling of exploited ecosystems. In Proceedings of the $6^{\text {th }}$ EUROSIM congress, Ljubjana, Slovenia, 2007.

[9] D. David and R. Courdier. Emergence as metaknowledge : refining simulation models through emergence reification. In Proceedings of ESM'2008, pages 25-27, Le Havre, France, 2008.

[10] J. Derveeuw. Artificial economics, agent-based methods in finance, game theory and their applications, volume 564 of Lecture notes in economics and mathematical system, chapter Market dynamics and agents behaviors : a computational approach, pages 15-26. Springer Berlin Heidelberg, 2006.

[11] M. Dorigo, V. Maniezzo, and A. Colorni. Ant system : optimization by a colony of cooperating agents. IEEE Transactions on Systems, Man, and Cybernetics, 26(1):29-41, 1996.

[12] A. Drogoul, D. Vanbergue, and T. Meurisse. Multi-agent based simulation : where are the agents? In Proceedings of $M A B S$, Lecture notes in computer science, pages 89-104. Springer Verlag, 2002.

[13] M.S. El hmam, H. Abouaissa, D. Jolly, and A. Benasser. Macro-micro simulation of traffic flow. In Proceedings of the12th IFAC Symposium on Information Control Problems in Manufacturing (INCOM06), pages 351356, Saint-Etienne, France, 17-19 mai 2006.

[14] J. Ferber and O. Gutknecht. A meta-model for the analysis and design of organizations in multi-agent systems. In Proceedings of the Third International Conference on Multi-Agent Systems (ICMAS98), pages 128-135, 1998. 
[15] Jacques Ferber. Les systèmes multi-agents : vers une intelligence collective. InterEditions, 1995.

[16] Tim Finin and Yannis Labrou andand James Mayfield. Software agents, chapter KQML as an agent communication language. MIT Press, Cambridge, 1997.

[17] D. Gross and B. McMullin. Is it the right ansatz? Artificial life, $7: 355-365$, 2001.

[18] Z. Guessoum, M. Ziane, and N. Faci. Monitoring and organizational-level adaptation of multi-agent systems. In ACM Press, editor, Proceedings of AAMAS'04, pages 514-521, 2004.

[19] Mahdi Hannoun, Olivier Boissier, Jaime Simão Sichman, and Claudette Sayettat. Moise : Un modèle organisationnel pour la conception de systèmes multi-agents. In Actes des JFIADSMA, pages 105-118, 1999.

[20] G. Kaminka, D. Pynadath, and M. Tambe. Monitoring teams by averhearing : a multi-agent planrecognition approach. Journal of Artificial Intelligence, $17: 83-135,2002$.

[21] A. Koestler. The ghost in the machine. Hutchinson, 1967.

[22] M. I. Marchenko. Medicolegal relevance of cadaver entomofauna for the determination of the time of death. Forensic Science International, $120: 89$ 120, 2001.

[23] H. R. Maturana and F. J. Varela. Autopoiesis and cognition the realization of the living, volume 42 of Boston Studies in the Philosophy of Science. Springer Berlin Heidelberg, 1980.

[24] S. McGregor and C. Fernando. Levels of description : a novel approach to dynamical hierarchies. Artificial life, 11(4) :459-472, 2005.

[25] F. Michel, G. Beurier, and J. Ferber. The turtlekit simulation platform : application to complex systems. In Proceedings of the First International Conference on Signal-Image Technology and Internet Based Systems, pages 122-127, 2005.

[26] G. Morel, H. Panetto, M.B. Zaremba, and F. Mayer. Manufacturing enterprise control and management system engineering : paradigms and open issues. IFAC Annual Reviews in Control, 27(2) :199-209, 2003.

[27] Edgar Morin. La méthode Tome 1. Editions du Seuil, 1977.

[28] G. Morvan, D. Jolly, A. Veremme, D. Dupont, and D. Charabidze. Vers une méthode de modélisation multi-niveaux. In Actes de la rème Conférence de Modélisation et Simulation MOSIM, Paris, France, 2008.

[29] Gildas Morvan, Daniel Jolly, Daniel Dupont, and Philippe Kubiak. A decision support system for forensic entomology. In Proceedings of the $6^{\text {th }}$ EUROSIM congress, Ljubjana, Slovenia, 2007.

[30] R. Pannequin, A. Thomas, and G. Morel. Proposition d'un environnement d'évaluation pour la mise en oeuvre d'un pilotage par le produit. In Actes de la rème Conférence de Modélisation et Simulation MOSIM, Paris, France, Paris, March29-April2 2008. 
[31] P. Ricordel and Y. Demazeau. Engineering Societies in the Agents World, chapter From analysis to deployment : a multi-agent platform survey, pages 93-105. Lecture notes in computer science. Springer Berlin Heidelberg, 2000.

[32] T. C. Schelling. Dynamic models of segregation. Journal of mathematical sociology, 1, 1971.

[33] R. Seifert, M.G. Kay, and J.R. Wilson. Evaluation of agv routing strategies using hierarchical simulation. In Proceedings of the Winter Simulation Conference (WSC), pages 850-856, Arlington, VA, USA, 1995.

[34] R. E. Stinner, A. P. Gutierrez, and G. D. Butler Jr. An algorithm for temperature-dependant growth rate simulation. The Canadian Entomologist, 106 :519-524, 1974.

[35] S. Terzi, J. Cassina, and H. Panetto. Development of a metamodel to foster interoperability along the product lifecycle traceability. In Proceedings of the IFIP/ACM SIGAPP INTEROP-ESA conference, Interoperability of Enterprise Software and Applications, pages 1-11, Geneva, Switzerland, February 23-25 2005. Springer.

[36] D. Trentesaux, T. Berger, and Y. Sallez. Product \& resource driven control of allocation and routing processes in fms. In Actes de la rème Conférence de Modélisation et Simulation MOSIM, Paris, France, 2008.

[37] J-P. Treuil, A. Drogoul, and J-D. Zucker. Modélisation et simulation à base d'agents. Dunod, 2008.

[38] K. Ueda, T. Kitob, and N. Fujii. Modeling biological manufacturing systems with bounded-rational agents. 2006.

[39] K. Ueda, A. Markusb, L. Monostorib, H.J.J. Kalsc, and T. Arai. Emergent synthesis methodologies for manufacturing. CIRP Annals - Manufacturing Technology, 50(2) :535-551, 2001.

[40] K. Ueda, J. Vaario, and K.H. Ohkura. Modelling of biological manufacturing systems for dynamic reconfiguration. 1997.

[41] H. Van Brussel. Holonic manufacturing systems, the vision matching the problem. In in Proc. of First European Conf. on Holonic Manufacturing Systems, 1994.

[42] H. Van Brussel, J. Wyns, P. Valckenaers, L. Bongaerts, and P. Peeters. Reference architecture for holonic manufacturing systems : Prosa. Computers in Industry, 37(3) :255-274, 1998.

[43] Francisco J. Varela, Evan Thompson, and Eleanor Rosch. L'inscription corporelle de l'esprit. Editions du Seuil, 1993.

[44] A. Veremme, D. Dupont, G. Morvan, D. Jolly, and D. Charabidze. Modélisation de la température d'un corps par automates cellulaires. In Actes de la rème Conférence de Modélisation et Simulation MOSIM, Paris, France, 2008. 
[45] A. Weber. Modélisation et gestion de flux par systèmes multiagents, application à un système d'aide à la décision en épidémiologie. $\mathrm{PhD}$ thesis, Ecole centrale de Lille, 2007.

[46] D. Wilkins, T. Lee, and P. Berry. Interactive execution monitoring of agent teams. Journal of Artificial Intelligence Research, 18 :217-261, 2003.

[47] M. Wooldridge. An introduction to multiagent systems. John Wiley \& Sons, 2002.

[48] T.D. Wyatt. Pheromones and animal behaviour : communication by smell and taste. Cambridge University Press, 2003. 